\title{
CT-detected solitary thyroid calcification: an important imaging feature for papillary carcinoma
}

This article was published in the following Dove Press journal:

OncoTargets and Therapy

13 October 2016

Number of times this article has been viewed

Tian-tian Yang ${ }^{1,2, *}$

Yong Huang,**

Xu-quan Jing ${ }^{1,2}$

Xiu-juan Gai, ${ }^{1,2}$

Wen-wu $\mathrm{Li}^{2}$

'School of Medicine and Life Sciences, University of Jinan-Shandong Academy of Medical Sciences, ${ }^{2}$ Department of Radiology, Shandong Cancer Hospital affiliated to Shandong University, Jinan, Shandong Province, People's Republic of China

*These authors contributed equally to this work
Correspondence: Wen-wu Li Department of Radiology, Shandong Cancer Hospital affiliated to Shandong University, No 440 Jiyan Road, Jinan, Shandong Province 250I I7, People's Republic of China

Tel +8653 I 67626582

Fax +8653167626582

Email Iwwzlm@sina.com
Purpose: To evaluate computed tomography (CT) detection of solitary thyroid calcification for identifying thyroid papillary carcinoma and to determine whether the predictive ability changes when the size increases after enhancement.

Materials and methods: CT scans on all 96 patients with thyroid nodules who underwent both enhanced CT examination of neck and thyroidectomy from 2014 to 2016 in the Shandong Cancer Hospital affiliated to Shandong University were reviewed. The cases without calcification and the cases with peripheral calcification, multiple coarse calcifications, or punctate calcification were excluded. Imaging features, including location and size of the lesions, were reviewed on plain and contrast-enhanced CT. The patients were grouped by histological results. The comparisons were evaluated by using Fisher's exact test and binary logistic regression.

Results: The study population consisted of 96 patients (74 females, 22 males; mean age $49.8 \pm 11.3$ years). Papillary thyroid carcinoma was observed in both solitary calcified thyroid nodules $(85.4 \%)$ and solely coarse calcifications surrounded by low-density focus $(58.2 \%)$. The difference was significant $(P=0.006)$. Of 64 patients with an amplification of lesions after contrast enhancement, $58(90.6 \%)$ were diagnosed with a malignant lesion. At the same time, of the 32 patients with no increase in size, 10 (31.2\%) were diagnosed with carcinoma and $22(68.8 \%)$ with nodular goiter. This difference was significant $(P<0.001)$, and after binary logistic regression, increasing size was an independent risk factor for cancer.

Conclusion: Solitary calcified thyroid nodules detected on CT represent a high risk for papillary thyroid carcinoma, especially when the size of the lesions increases after contrastenhanced CT.

Keywords: computed tomography, solitary calcified thyroid nodule, papillary thyroid carcinoma, size

\section{Introduction}

Thyroid nodules are common. Approximately $4 \%-7 \%$ of the population have palpable thyroid nodules, ${ }^{1}$ and the prevalence of nonpalpable nodules is even higher. ${ }^{2}$ Ultrasonography (US), Doppler US, fine-needle aspiration, and computed tomography (CT) are all used for inspection of the thyroid, of which US is the most frequently used. ${ }^{1,3-5}$ However, US is easily affected by the subjectivity of the various operators. ${ }^{2}$ Furthermore, the use of Doppler US to define blood supply present in thyroid nodules for diagnostics is disputed. Some investigators think that rich vasculature in nodules plays a role in elucidating the characteristics, ${ }^{2,6}$ whereas others consider that it does not provide supplementary information. ${ }^{7,8}$ Fine-needle aspiration is probably unnecessary in diffusely enlarged glands with multiple nodules, noninvading lesions to normal parenchyma, ${ }^{9}$ and nodules with coarse calcifications, which are difficult to penetrate by needle. ${ }^{10}$ Reports about thyroid nodules detected on CT are rare. It is generally accepted 
that CT may underestimate the number of nodules compared with ultrasound. A study also showed that no CT features can reliably differentiate benign thyroid nodules from malignant ones. ${ }^{11}$ However, CT provides unique advantages for evaluation of thyroid gland tumors, particularly for advanced lesions with suspicion of extracapsular extension, ${ }^{3}$ or metastatic cervical lymph nodules compared to the limited number of images available from sonographic examinations, ${ }^{12,13}$ or incidentally detected lesions of the thyroid gland in neck or chest CT examinations. ${ }^{14}$ In clinical practice, CT examination is feasible for the diagnosis of thyroid nodules.

Calcification is a common phenomenon in both benign and malignant thyroid nodules, ${ }^{15,16}$ and the incidence of calcification in malignant nodules is higher. ${ }^{17,18}$ Many studies have assessed various calcifications for their diagnostic value in benign and malignant nodules. ${ }^{5,10,15-18}$ Solitary calcified thyroid nodules are examples of single coarse calcifications without visualization of the surrounding low-density focus whose density is lower than the normal thyroid gland tissue. ${ }^{17}$ Kwak et al ${ }^{19}$ reported that cases that show solitary calcified nodules without soft tissue involvement can be diagnosed as thyroid papillary carcinoma on ultrasound, and $\mathrm{Ma}$ and $\mathrm{Li}^{20}$ also showed that malignant pathology was present in $40 \%$ of five cases on ultrasound. Therefore, solitary calcified thyroid nodules should not be ignored. CT imaging studies of these nodules have not been reported. The sensitivity of ultrasound for the diagnosis of calcification is $82 \%-96 \%$, which is a little larger than the $75 \%$ sensitivity of CT scans. However, in preoperative ultrasonographic diagnosis of calcified nodules, more than half the nodules were observed to be without calcification, whereas the preoperative CT diagnosis of calcified nodules is usually consistent with the pathology. On the other hand, the specificity of ultrasound is lower. Han et $\mathrm{al}^{21}$ reported that the reason for this low specificity is that the fiber composition of these colloid nodules was characterized by clusters of high-echo-like calcification. Furthermore, posterior attenuation disturbs identification of the lesion's constitution behind annular or coarse calcification, so the application in ultrasound has limits. On the other hand, CT has unique advantages in identifying solitary calcified thyroid nodules. In the present report, we assess the association between solitary calcified thyroid nodules detected on CT and papillary carcinoma as well as the changes of lesions size, to study prediction function of CT in thyroid disease.

\section{Materials and methods Study population}

A retrospective review was performed on all 820 patients who underwent both CT examination of neck and thyroidectomy in Shandong Cancer Hospital affiliated to Shandong University from July 2014 to July 2016. We excluded 344 cases without calcification, 194 with punctate calcification, 118 with peripheral calcification, and 68 with multiple coarse calcifications (Figure 1). Finally, we identified 41 cases with solitary calcified nodules and 55 cases with solely coarse calcification surrounded by a low-density focus for inclusion in the study group. The final study population consisted of 96 cases ( 74 women and 22 men; range $23-75$ years; mean

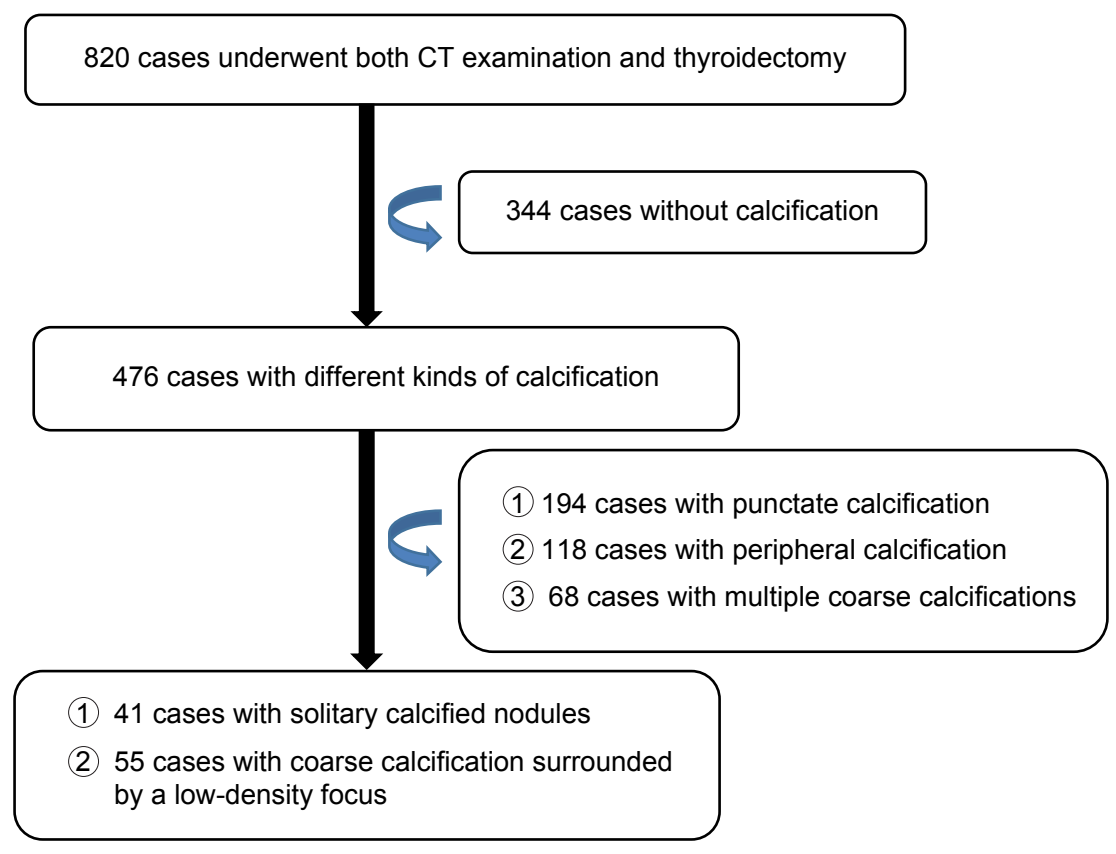

Figure I The flowchart of patients' selection.

Abbreviation: CT, computed tomography. 
age $49.8 \pm 11.3$ years). The study was approved by the ethics committee of the Shandong Cancer Prevention and Treatment Research which deemed patient consent was not required due to the retrospective nature of the study.

\section{Methods}

All the 820 patients underwent contrast-enhanced CT scans of the neck. All CT scans were obtained using a highspeed 128 slice spiral CT scanner (GE Medical Systems, Waukesha, WI, USA). The scanning parameters were: 120 $\mathrm{kV}, 250 \mathrm{mAs}, 32 \times 0.625$ collimation, 0.75 seconds rotation time, 1.7 seconds cycle time, $2.5 \mathrm{~mm}$ cross-sectional thickness, and $2.5 \mathrm{~mm}$ cross-sectional distance. Before scanning, patients were told to breathe calmly and not to swallow. The patients were placed in supine position with the neck fully exposed, and the scanning range was at least from pharynx oralis to the upper edge of the aortic arch. The contrast medium used was Omnipaque $(300 \mathrm{mg} / \mathrm{L}$; Amersham Health, Cork, Ireland) with $80-100 \mathrm{~mL}$ injected via the median cubital vein at a rate of $2.7-3.0 \mathrm{~mL} / \mathrm{s}$. Dual-phase enhanced scanning was performed at 30 and 70 seconds after contrast injection.

\section{Image analysis}

The images were converted to $1 \mathrm{~mm}$ cross-sectional thickness and $1 \mathrm{~mm}$ of cross-sectional distance through imaging postprocessing techniques and then independently assessed by two radiologists (WWL and $\mathrm{YH}$ ) with 30-40 years of experience who were blinded to the results of pathology recorded on the Picture Archiving and Communication Systems Workstation. If the radiologists disagreed, they made the final decision through further consultations and dialogue. The following CT parameters were evaluated: location and the size of lesions before and after contrast enhancement. Also, the standard of size is region of interest (ROI). We chose the layer of maximal size of calcification, before and after enhancement. When $\mathrm{ROI}_{2}-\mathrm{ROI}_{1}>10 \% \mathrm{ROI}_{1}$, the size increased after contrast enhancement.

\section{Pathology}

All 820 patients had a conclusive pathological diagnosis confirmed by senior pathologists. All thyroid specimens were fixed in $10 \%$ neutral formalin. Tissue sections of $2 \mathrm{~mm}$ thickness were stained with hematoxylin and eosin to ensure that the calcifications were embedded in the specimen.

\section{Statistical analysis}

Statistical analysis of the results was performed with SPSS 17.0 (SPSS Inc., Chicago, IL, USA). Fisher's exact test was used to compare the difference in age, sex, the incidence of a surrounding low-density focus, and the incidence of increasing size. Binary logistic regression was used to assess the association between the histopathologic diagnosis and low-density focus or increasing size. A $P$-value $<0.05$ was considered to be statistically significant.

\section{Results}

During the study period, 820 patients underwent both thyroidectomy and preoperative contrast-enhanced CT. In all, there were 280 papillary carcinomas, 16 follicular carcinomas, five medullary carcinomas, 14 undifferentiated carcinomas, 301 nodular goiters, 78 thyroid adenomas, 35 cystic degeneration of thyroid adenomas, and 91 inflammatory nodules. The rate of malignancy in cases without calcification, with peripheral calcification, with punctate calcification, with solitary calcified nodules, and with coarse calcification surrounded by a low-density focus were $11.8 \%$, $20.2 \%, 39.8 \%, 85.4 \%$, and $43.3 \%$, respectively. A total of 96 patients were finally included in the study, with an age range between 23 and 75 (mean age 49.8土11.3) years. Among 66 patients older than 45 years, 46 (69.7\%) were diagnosed with papillary thyroid carcinoma, whereas in 30 patients younger than 45 years, $28(93.3 \%)$ had malignant pathology. Among 96 patients, the male-to-female ratio was 1:3.4. Of the 22 males, $21(95.5 \%)$ were found to have papillary thyroid carcinoma. Of the 74 females, 53 (71.6\%) were diagnosed with papillary thyroid carcinoma. In the group of 41 patients with a solitary calcified nodule, $35(85.4 \%)$ had malignant pathology, whereas $32(58.2 \%)$ of cases were diagnosed with papillary carcinoma in the group of 55 patients with solely coarse calcification surrounded by a low-density focus. In all the 96 cases, 58 (89.2\%) had a diagnosis of papillary carcinoma in the 64 patients with increasing size, whereas the malignancy rate in the 32 cases without increasing size was $31.3 \%$. The $P$-values were 0.01 for age, and 0.02 for sex, 0.006 for low-density focus, and 0.001 for increasing size. The differences were significant (Table 1).

As indicated by the binary logistic regression analysis, there were significant differences in the incidence of size increase $(P=0.001$, odds ratio $=0.056$; Table 1$)$. The incidence of size increase had area under the receiver operating characteristic curve of 0.805 (Figure 2).

\section{Discussion}

The malignancy rate of solitary calcified thyroid nodules detected on CT was $85.4 \%$, and the pathologies were all papillary thyroid carcinomas. Especially when the size of 
Table I Patient characteristics

\begin{tabular}{lllll}
\hline Subgroup & Malignant & Benign & P-value & OR \\
\hline Age, years & & & 0.01 & - \\
$\quad \geq 45$ & 46 & 20 & & \\
$\quad<45$ & 28 & 2 & & \\
Sex & & & 0.02 & - \\
$\quad$ Female & 53 & 21 & & \\
$\quad$ Male & 21 & 1 & & \\
Incidence of low-density focus & & & 0.006 & - \\
$\quad$ Yes & 32 & 23 & & \\
$\quad$ No & 35 & 6 & & \\
Incidence of increasing size & & & 0.001 & 0.056 \\
$\quad$ Yes & 58 & 6 & & \\
No & 10 & 22 & & \\
\hline
\end{tabular}

Abbreviation: OR, odds ratio.

the lesions increased after contrast-enhancement CT, the pathologies were almost malignant.

Thyroid carcinoma is a malignant tumor with rapidly increasing incidence, and it is also one of the most common malignant tumors of the endocrine system. Most thyroid nodules are benign, but small proportions of such nodules are malignant. According to the survey conducted by World Health Organization, the cure rate of thyroid carcinoma is approximately $85 \%$. So, early diagnosis and treatment is very important, and the main treatment modality is surgery. Accordingly, the unnecessary operation rate of thyroid nodules has also increased rapidly. Lifelong thyroid replacement therapy adds to the burden of the patients and their families. Additionally, lack of consensus, specification, surgical procedure, and recurrence monitoring augment the crisis.

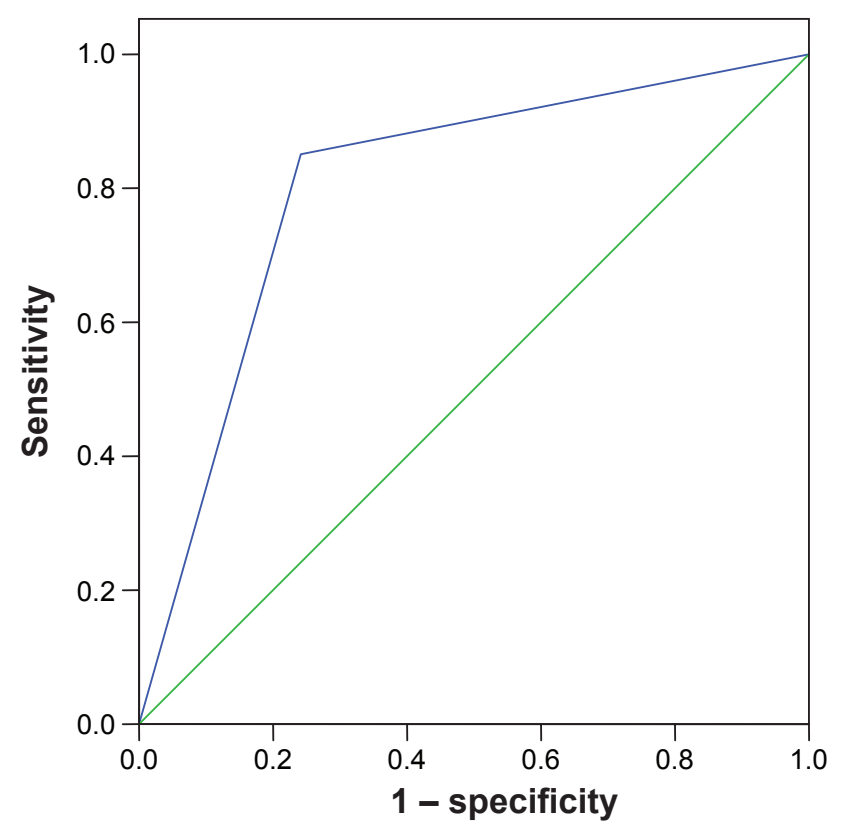

Figure 2 ROC curve of diameters.

Abbreviation: ROC, receiver operating characteristic.
In our study, the relative risk for malignancy was higher in the younger patients than in the older patients $(P<0.05)$. This is in accordance with the previous report of Kakkos et al. ${ }^{15}$ In addition, our study showed significant differences between males and females $(P=0.02)$, similar to the results of previous study. ${ }^{15}$

Calcification is common in thyroid tissue. Wu et a ${ }^{14}$ reported that calcification is present in $35 \%$ of thyroid nodules in CT. The patterns of calcification in CT include peripheral calcification, coarse calcifications $(>2 \mathrm{~mm})$, and punctate calcification. The punctate calcification consists of single punctate calcification and multiple punctuate calcification. Previous studies have reported various patterns of calcification. Frates et al ${ }^{9}$ reported that the presence of any calcification within the nodule increases the likelihood of malignancy.

Peripheral calcification patterns include "egg-shell"like, rim-like, or curvilinear calcification located around the nodule. ${ }^{14}$ It is one type of dystrophic calcifications. However, when compared with nodules without calcification, the presence of peripheral calcification did not show obvious increase in the relative risk of malignancy, ${ }^{17,21}$ but was associated with a multinodular goiter. Kim et al ${ }^{22}$ deduced that peripheral calcification is sometimes associated with malignancy when a peripheral halo is noted and disruption disappears in sonography.

Punctate calcification is defined as a single or multiple intranodular microcalcification that measures $\leq 2 \mathrm{~mm}$ in diameter. Shetty et $\mathrm{a}^{23}$ found that the presence of punctate calcification on CT correlated to the presence of microcalcifications on sonography, and this statement is in accord with the report by Ishigaki et al. ${ }^{3}$ Meanwhile, some previous reports showed that microcalcification was an independent risk factor for malignancy. ${ }^{6,15,17,21}$ Shetty et $\mathrm{al}^{23}$ reported that the incidence of punctate calcification may increase suspicion, but no significant correlation existed between punctate calculation on CT and malignant or potentially malignant histology. Wu et $\mathrm{al}^{14}$ reported that nodules with multiple punctate calcifications (79\%) were more likely to be malignant than nodules with a single punctate calcification (58\%). Wu et al ${ }^{14}$ also indicated that when solitary calcified nodule occurs with multiple punctate patterns, resection is strongly recommended.

Coarse calcification presented as large, nodular, or amorphous opaque structures that measured $>2 \mathrm{~mm}$ in diameter. ${ }^{14}$ It is also a kind of dystrophic calcification. Coarse calcification has been reported to be helpful in the diagnosis of malignancy, and its presence increases the likelihood of cancer almost twofold when compared with the malignant nodules without calcification. ${ }^{15,22}$ 
Studies about solitary calcified nodules are rare. In our study, of the 41 cases with solitary calcified nodules, $35(85.4 \%)$ were diagnosed as carcinoma. The ratio was larger compared with the result by $\mathrm{Ma}$ and $\mathrm{Li}^{20}(40 \%)$, but a little smaller compared with Kwak's results (100\%). We carried on our study to further elaborate the difference between the benign and malignant lesions. Although our case series was smaller, it is meaningful when compared to other's results. Wu et a $1{ }^{14}$ showed that thyroid calcification found on preoperative $\mathrm{CT}$ may represent an increased risk for thyroid malignancy, and the malignancy rate of peripheral calcification, coarse calcification, single punctate calcification, multiple punctate calcification, and solitary calcified nodules was $22 \%, 21 \%, 58 \%, 79 \%$, and $83 \%$, respectively. This result is similar to our findings. Kwak et al ${ }^{19}$ showed that eleven cases with solitary calcified nodules all had microcalcifications. Microcalcification is closely associated with papillary carcinoma, so our results have clinical significance.

In our study, we found that the increase in lesion size was closely related to malignant pathologies results. Previously, there were no reports explaining the change in the size before and after the contrast-enhanced CT. We also found that window width and window level would affect the size of lesions. As shown in Figure 3, the sizes of lesions before and after enhancement in bone window craniotomy were fixed. This phenomenon explained the reason that calcification

A
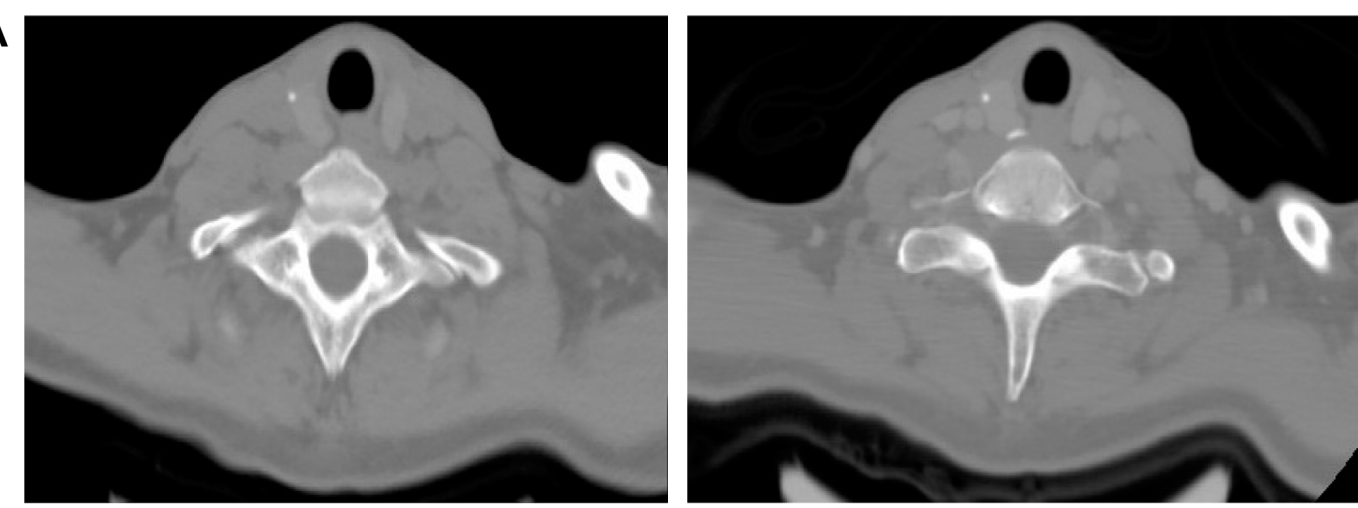

B
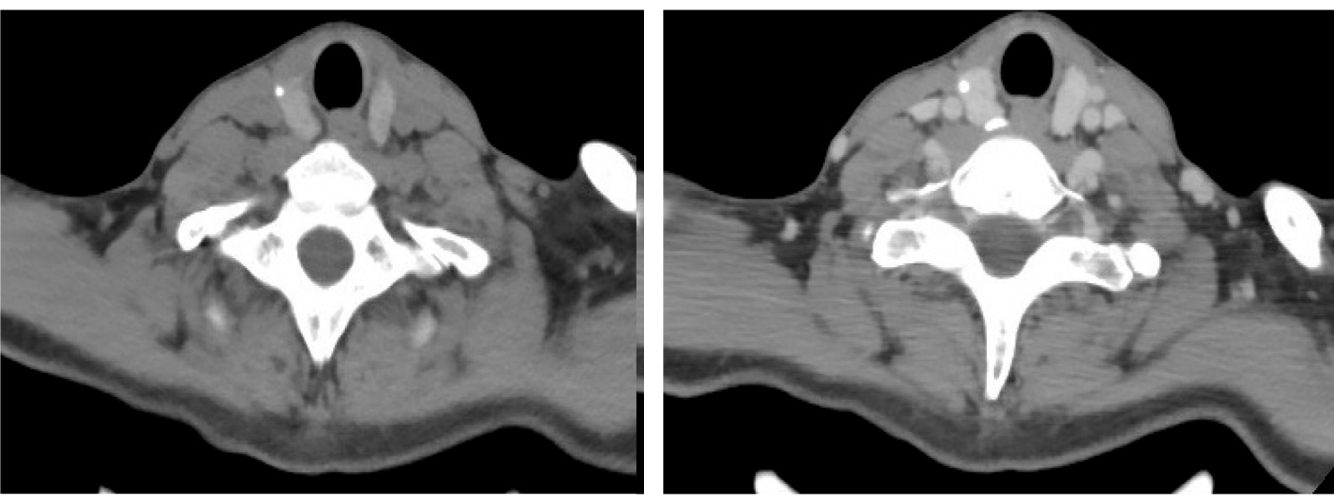

C
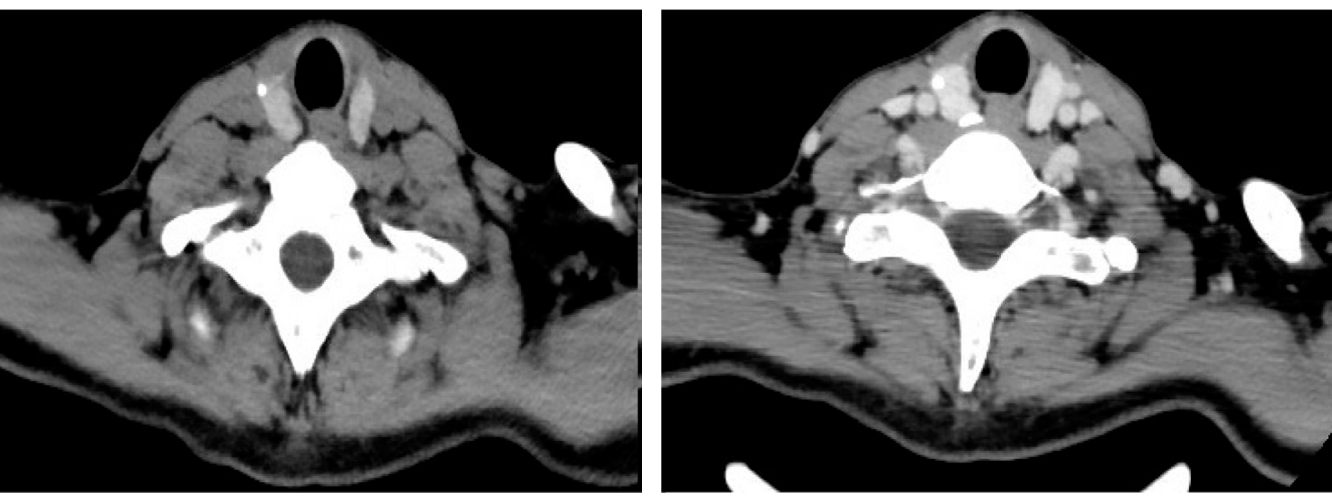

Figure 3 A 48-year-old female presented to our hospital because of a thyroid nodule found incidentally on physical examination, and the pathology was papillary carcinoma.

Notes: The size of lesion before and after enhancement in bone window craniotomy was fixed. This phenomenon explained that the size of calcification has not actually changed. But the amplification is larger in abdomen window than in mediastinum window. (A) Bone window, (B) mediastinum window, and (C) abdomen window. 
remains virtually unchanged. But the amplification is larger in abdomen windows than in mediastinum windows. Although we have not yet determined the mechanism for the increase in the size of lesions after enhancement, we infer that mesenchyme is rich in vessels surrounding calcification, which constitutes the enlarged part after enhancement. But the mesenchyme is too thin to be distinguished on plain CT scan. Han et $\mathrm{al}^{25}$ indicated that malignant nodules were rich in blood supply compared with benign nodules, especially microcarcinomas, and also malignant nodules usually have less fibrous tissue and show invasive growth tendency. Certainly, a study ${ }^{10}$ suggested that contrast-enhanced CT was not appropriate if the radioactive iodine was administered, because the iodide ions may influence the final therapeutic effects. Mishra et $\mathrm{al}^{26}$ studied the relationship between iodinated contrast media used in contrast-enhanced CT and radioiodine therapy. The majority of contrast agents are water-soluble, which helps avoid the phenomenon observed with lipophilic contrast agents, ie, storage in adipose tissue for a long time. Previous studies ${ }^{27,28}$ showed that iodine may be retained for many years after contrast-enhanced $\mathrm{CT}$. A recent study ${ }^{25}$ have reported that the interval from contrast-enhanced CT to surgery is becoming shorter. The half-life of iodine is 10 hours, and the excess administered iodine is excreted in the urine quickly. Nonlipophilic contrast cannot be retained in extracellular fluids; therefore, iodine levels should revert to previous equilibrium levels soon after any contrast study. Other higher levels will occur only if there is further iodine contamination. ${ }^{24}$ Therefore, performing contrast-enhanced $\mathrm{CT}$ is reasonable. Zhu et $\mathrm{al}^{10}$ indicated that enhanced CT does not provide more available information than a CT scan for solitary calcified nodules, but it increases the cost and radiation exposure. However, Han et $\mathrm{al}^{25}$ showed clearly that the information on the boundary of tumor is clear in unenhanced or contrast-enhanced scans, but has limited value in differentiating benign and malignant nodules. It is generally understood that irregular boundaries are important features of thyroid malignancies. This discovery illustrated the importance of contrast-enhanced CT. On the basis of the results of our study, it can be concluded that contrast-enhanced CT is important for differentiating benign and malignant nodules.

\section{Limitations}

There are several limitations to our study. First, the evaluation of cases was retrospective, and so selection bias was unavoidable. The importance of solitary calcified thyroid nodules has not been realized, and the risk of nodules with tumor around a single punctate calcification was considered not high. We could not rule out the possibility that there were some cases undergoing thyroidectomy without preoperative CT or cases undergoing preoperative CT without thyroidectomy. Second, we may not have observed some solitary calcified thyroid nodules even in $1 \mathrm{~mm}$ section thickness of the CT images. Because the change in the neck structure is usually large, the quality of images may also influence the final observations. Third, we did not evaluate the CT features, because the lesions were generally small, and it is difficult to describe their shapes, margins, and so on.

\section{Conclusion}

To summarize, although US is the most commonly used technique in thyroid examination, CT is increasingly utilized because of its unique advantages in large or retrosternal goiter and malignant thyroid lesions with suspicion of extracapsular extension or cervical metastasis. ${ }^{14}$ When the CT showed solitary calcified thyroid nodules, especially with the increasing of lesion size after enhancement, we found a very high risk of malignancy.

\section{Disclosure}

The authors report no conflicts of interest in this work.

\section{References}

1. Seiberling KA, Dutra JC, Grant T, Bajramovic S. Role of intrathyroidal calcifications detected on ultrasound as a marker of malignancy. Laryngoscope. 2004;114(10):1753-1757.

2. Chan BK, Desser TS, McDougall IR, Weigel RJ, Jeffrey RB Jr. Common and uncommon sonographic features of papillary thyroid carcinoma. J Ultrasound Med. 2003;22(10):1083-1090.

3. Ishigaki S, Shimamoto K, Satake H, et al. Multi-slice CT of thyroid nodules: comparison with ultrasonography. Radiat Med. 2004;22(5): 346-353.

4. Cooper DS, Doherty GM, Haugen BR, et al. Management guidelines for patients with thyroid nodules and differentiated thyroid cancer. Thyroid. 2006;16(2):109-142.

5. Yoon DY, Chang SK, Choi CS, et al. The prevalence and significance of incidental thyroid nodules identified on computed tomography. J Comput Assist Tomogr. 2008;32(5):810-815.

6. Papini E, Guglielmi R, Bianchini A, et al. Risk of malignancy in nonpalpable thyroid nodules: predictive value of ultrasound and color-Doppler features. J Clin Endocrinol Metab. 2002;87(5):1941-1946.

7. Iannuccilli JD, Cronan JJ, Monchik JM. Risk for malignancy of thyroid nodules as assessed by sonographic criteria: the need for biopsy. J Ultrasound Med. 2004;23(11):1455-1464.

8. Wienke JR, Chong WK, Fielding JR, Zou KH, Mittelstaedt CA. Sonographic features of benign thyroid nodules: interobserver reliability and overlap with malignancy. J Ultrasound Med. 2003;22(10): 1027-1031.

9. Frates MC, Benson CB, Charboneau JW, et al. Management of thyroid nodules detected at US: Society of Radiologists in Ultrasound consensus conference statement. Ultrasound Q. 2006;22(4):231-238; discussion 239-240.

10. Zhu D, Chen W, Xiang J, Han Z. Diagnostic value of CT artifacts for solitary coarse calcifications in thyroid nodules. Int J Clin Exp Med. 2015;8(4):5800-5805. 
11. Hoang JK, Riofrio A, Bashir M, Kranz PG, Eastwood JD. High variability in radiologists' reporting practices for incidental thyroid nodules detected on CT and MRI. Am J Neuroradiol. 2014;35(6):1190-1194.

12. Kim E, Park JS, Son KR, Kim JH, Jeon SJ, Na DG. Preoperative diagnosis of cervical metastatic lymph nodes in papillary thyroid carcinoma: comparison of ultrasound, computed tomography, and combined ultrasound with computed tomography. Thyroid. 2008;18(4): 411-418.

13. Soler ZM, Hamilton BE, Schuff KG, Samuels MH, Cohen JI. Utility of computed tomography in the detection of subclinical nodal disease in papillary thyroid carcinoma. Arch Otolaryngol Head Neck Surg. 2008; 134(9):973-978.

14. Wu CW, Dionigi G, Lee $\mathrm{KW}$, et al. Calcifications in thyroid nodules identified on preoperative computed tomography: patterns and clinical significance. Surgery. 2012;151(3):464-470.

15. Kakkos SK, Scopa CD, Chalmoukis AK, et al. Relative risk of cancer in sonographically detected thyroid nodules with calcifications. J Clin Ultrasound. 2000;28(7):347-352.

16. Khoo ML, Asa SL, Witterick IJ, Freeman JL. Thyroid calcification and its association with thyroid carcinoma. Head Neck. 2002;24(7): $651-655$.

17. Kim BK, Choi YS, Kwon HJ, et al. Relationship between patterns of calcification in thyroid nodules and histopathologic findings. Endocrine J. 2013;60(2):155-160.

18. Liu W, Dong X, Zhu C, et al. Association between computed tomography-detected calcification and thyroid carcinoma. Neoplasma. 2015;62(4):641-645.

19. Kwak JY, Kim EK, Son EJ, et al. Papillary thyroid carcinoma manifested solely as microcalcifications on sonography. AJR Am J Roentgenol. 2007;189(1):227-231.
20. Ma Y, Li M. The correlation analysis between calcification type and distribution in thyroid carcinoma. Chin Imaging J Integr Tradit West Med. 2014;12(2):195-197.

21. Han ZJ, Gu Y, Lei ZK, et al. The value of ultrasound in the differential diagnosis of benign and malignant thyroid calcification nodule. Chin J Ultrasound Med. 2015;31(11):1045-1047.

22. Kim BM, Kim MJ, Kim EK, et al. Sonographic differentiation of thyroid nodules with eggshell calcifications. J Ultrasound Med. 2008;27(10): 1425-1430.

23. Shetty SK, Maher MM, Hahn PF, Halpern EF, Aquino SL. Significance of incidental thyroid lesions detected on CT: correlation among CT, sonography, and pathology. AJR Am J Roentgenol. 2006;187(5): 1349-1356.

24. Frates MC, Benson CB, Doubilet PM, et al. Prevalence and distribution of carcinoma in patients with solitary and multiple thyroid nodules on sonography. J Clin Endocrinol Metab. 2006;91(9):3411-3417.

25. Han ZJ, Shu YY, Lai XF, Chen WH. Value of computed tomography in determining the nature of papillary thyroid microcarcinomas: evaluation of the computed tomographic characteristics. Clin Imaging. 2013; 37(4):664-668.

26. Mishra A, Pradhan PK, Gambhir S, Sabaretnam M, Gupta A, Babu S. Preoperative contrast-enhanced computerized tomography should not delay radioiodine ablation in differentiated thyroid carcinoma patients. J Surg Res. 2015;193(2):731-737.

27. Costa A, Testori OB, Cenderelli C, Giribone G, Migliardi M. Iodine content of human tissues after administration of iodine containing drugs or contrast media. J Endocrinol Invest. 1978;1(3):221-225.

28. Spate VL, Morris JS, Chikos S, et al. Determination of iodine in human nails via epithelial neutron activation analysis. Radioanal Nucl Chem. 1998;195(1):236-271
OncoTargets and Therapy

\section{Publish your work in this journal}

OncoTargets and Therapy is an international, peer-reviewed, open access journal focusing on the pathological basis of all cancers, potential targets for therapy and treatment protocols employed to improve the management of cancer patients. The journal also focuses on the impact of management programs and new therapeutic agents and protocols on

\section{Dovepress}

patient perspectives such as quality of life, adherence and satisfaction. The manuscript management system is completely online and includes a very quick and fair peer-review system, which is all easy to use. Visit http://www.dovepress.com/testimonials.php to read real quotes from published authors. 